\title{
Emergency laparoscopic colorectal surgery
}

\author{
• Emrah Şahin, ๑ Ersin Gündoğan, ๑ Cüneyt Kayaalp \\ Department of Genaral Surgery, İnönü University Faculty of Medicine, Malatya, Turkey
}

\begin{abstract}
Introduction: The laparoscopic technique is increasingly used in colorectal surgery. However, in emergency cases, the use of the laparoscopic method is still limited. This was a study of the outcomes of laparoscopic surgery in emergency cases of colorectal disease at a single center.

Materials and Methods: The demographic data and perioperative findings of patients who underwent emergency laparoscopic colorectal surgery between July 2013 and January 2019 were retrospectively analyzed.

Results: An emergency laparoscopy was performed on a total of 14 of 658 (2.1\%) patients who underwent colorectal surgery. Eight (57.1\%) were male and the mean age was $55.2 \pm 21.6$ years. Conversion to open surgery was required in 5 cases (35.7\%). The mean number of lymph nodes removed from the patients operated on for tumors was $22.5 \pm 17.5$.

Conclusion: The results of this study suggest that the laparoscopic approach can be applied in emergency cases of colorectal disease in certain circumstances. However, the rate of conversion to open surgery was greater than for non-emergency laparoscopic surgery. Laparoscopy was most useful for colonoscopy perforations and some colonic obstructions.
\end{abstract}

Keywords: Colonoscopy; colorectal cancer; laparoscopy; perforation; tumor.

\section{Introduction}

Laparoscopy has been an important milestone in surgery with reduced postoperative pain, wound complications, hospital stay and return to early daily life. It was first used in colon surgery in $1991^{[1]}$ and has been used with increasing frequency so far. However, its use in emergency colorectal surgery did not follow the elective surgery trend. The main reasons are exploration restriction in the abdomen in case of intestinal obstruction and fear of inadequate tumor surgery. Presence of peritonitis and adhesions in emergency cases technically make the operations more challenging, additionally, requirement of experi- enced team and equipment limit laparoscopic approach in emergency cases.

The aim of this study was to examine the patients who underwent emergency laparoscopic colorectal surgery in our clinic.

\section{Materials and Methods}

Data from patients who underwent emergency laparoscopic colorectal surgery between July 2013 and January 2019 were retrospectively collected. Patients who underwent conversion were also included in the study. The demographic data (age, gender, ASA), operation types, length 
of stay, pathology results, reoperation requirements, morbidity and mortalities were evaluated. Data were collected and analyzed in Microsoft Excel 2013. Fisher's exact test was used to compare the categorized data statistically.

\section{Operation Technique}

The patients were operated in supine position. The first trocar was inserted under the umbilicus with open technique. Then, according to the pathology, the other trocars were entered in the left or right quadrant on midclavicular line spaced $8 \mathrm{~cm}$ apart. Surgical procedure was performed according to the primary diagnosis. One drain was placed in the pelvis and the operation was terminated. Laparotomy was performed from the median line in conversion patients.

\section{Results}

A total of 658 colorectal operations were performed in our clinic between July 2013 and January 2019. Of these, 501 them were elective surgeries and $243(48.5 \%)$ of these cases were done laparoscopically. 27 of these patients (11.1\%) required conversion.

During the same period, 157 patients underwent emergency colorectal surgery and 14 (8.9\%) of these patients underwent laparoscopic surgery. In 5 patients (35.7\%) who underwent laparoscopic surgery, conversion to open surgery was required. In emergency laparoscopy, conversion rate to open was higher than the elective laparoscopic colorectal surgeries $(\mathrm{p}=0.016)$.

Of the 14 patients who underwent laparoscopic emergency colorectal surgery, $8(57.1 \%)$ were male and the mean age was $55.2 \pm 21.6$ years. No patient had a previous history of abdominal surgery. Causes of emergency colorectal surgery were mechanical bowel obstruction $(10,71.4 \%)$ and acute ab-

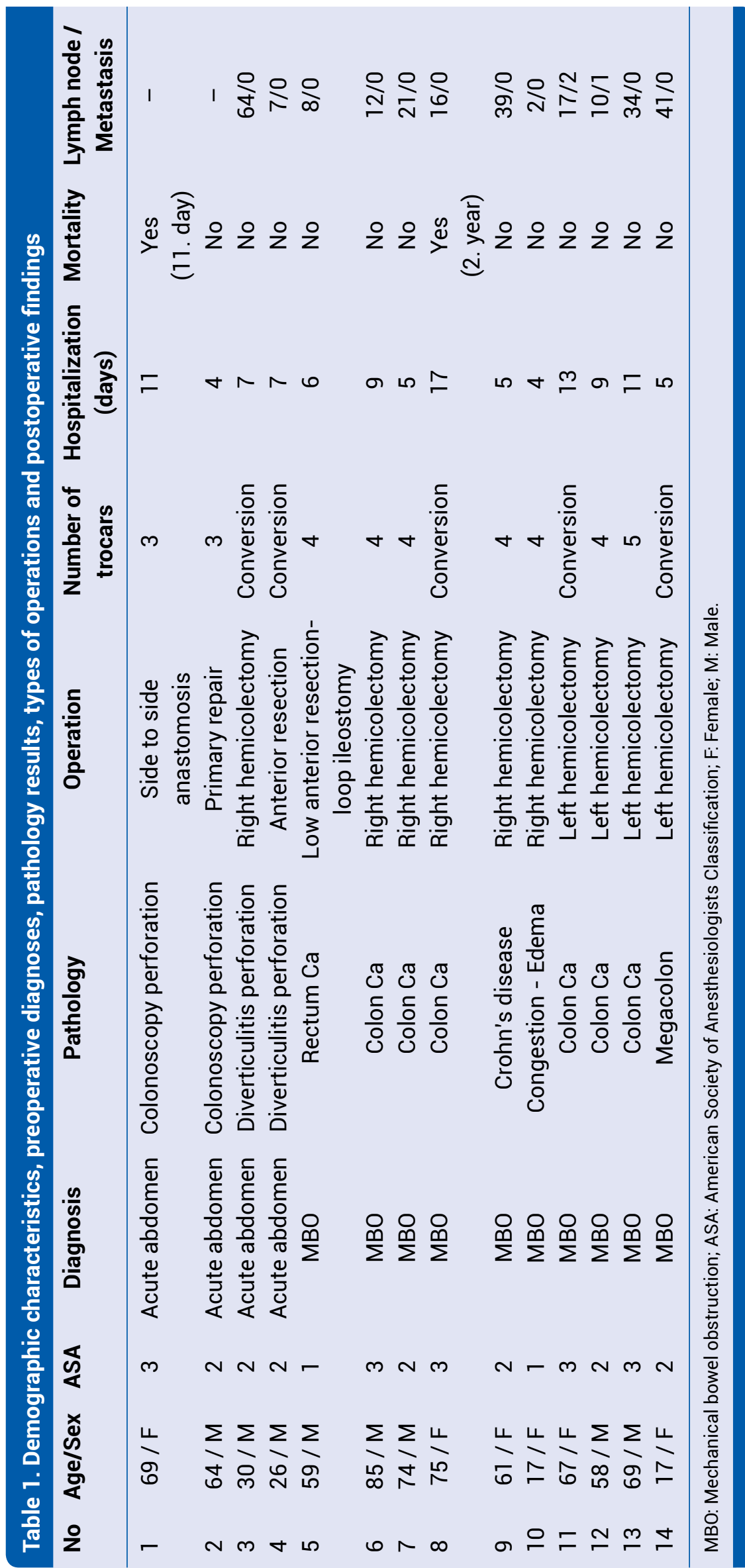


domen (4, 28.5\%). Two patients with mechanical bowel obstruction had hypertension as additional disease, one patient had ITP, and one patient had a cardiac pacemaker. Coronary artery disease and prostate cancer were also present in a patient with hypertension. One of the patients who presented with acute abdominal pain had hypertension as an additional disease and another patient had lung adenocarcinoma. The median ASA score of the patients were 2 (1-3). The median trocar number was 4 (3-5). Patients' demographics, preoperative and postoperative data were given in Table 1. The mean duration of hospitalization was $8.0 \pm 3.6$ days. In all malignant patients, the surgical margin was negative and the median retrived lymph nodes were 16 (2-64). No postoperative morbidity was observed in any patient but one patient died in the early postoperative period. This patient was operated because of perforation in the sigmoid colon during colonoscopy and peritoneal carcinomatosis was diagnosed during laparoscopy. This patient's biopsy revealed metastasis of lung adenocarcinoma. Since the perforation area occupied almost all of the lumen, side-by-side anastomosis was performed and the patient died due to cardiopulmonary insufficiency unrelated to abdominal problem on the $11^{\text {th }}$ postoperative day. The median followup period was 8 (2-65) months and none of the cancer patients had recurrence or metastasis.

\section{Discussion}

Despite the reported benefits of laparoscopy in the elective treatment of benign and malignant colorectal diseases,
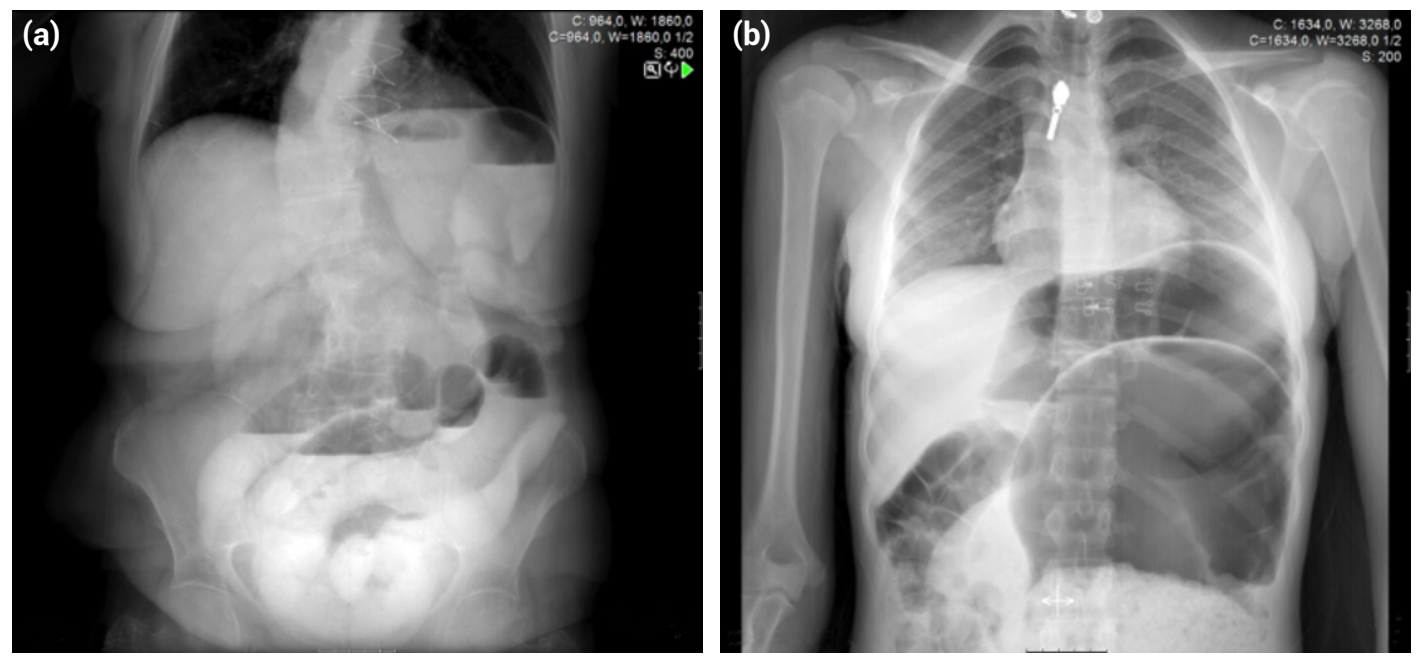

Figure 1. (a) Preoperative image, ileal perforation due to a mass in the cecum (Patient \# 8). (b) Preoperative image of toxic megacolon (Patient \# 14).
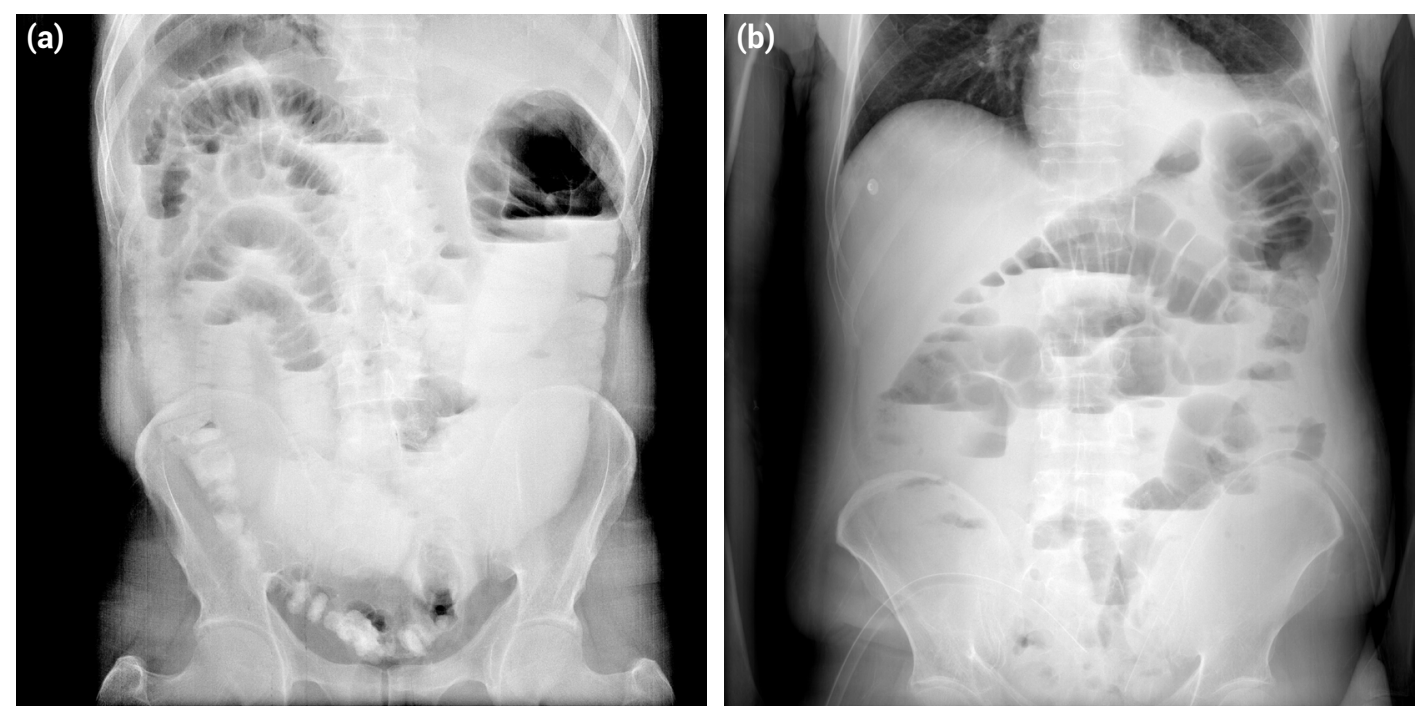

Figure 2. (a) Preoperative image, obstruction, due to a mass in the left colon (Patient \# 12). (b) Preoperative image, obstruction, due to a mass in the left colon (Patient \# 13). 
laparoscopic colectomy has not become widespread in emergency settings. The laparoscopic approach in acute colonic obstruction is still considered by many surgeons as an absolute contraindication. ${ }^{[2,3]}$ Some reasons to say; difficulty in creating a pneumoperitoneum, limited working area in the abdominal cavity, instability of the patient, difficulty of handling of the dilated intestines and experienced surgeon requirements. ${ }^{[4,5]}$ The reasons of our conversion were dilatation of the intestine, technical difficulties due to narrowing in the abdominal cavity, inability to form enough pneumoperitoneum, difficulty in proper surgical resection because of the tumor's adherence to surrounding tissues.

While the mortality rate associated with elective colonic resection is less than $5 \%$, this rate can increase to $23 \%$ following emergency colorectal resections. ${ }^{[6]}$ We did not do a comparative study of emergency and elective laparoscopic colorectal surgeries. One of the limitations of our study was the variety of surgical indications and etiologies. There were also a limited number of patients in each group. Literature data on emergency laparoscopic right hemicolectomy in the right colon obstruction is lacking. There are controversial aspects in the left colon obstruction, such as endoscopic colonic stents before surgery. Previous reports demonstrated that laparoscopic left hemicolectomy can be performed in only one-fourth of these patients. ${ }^{[7,8]}$ Previous studies on the role of laparoscopic colectomy in inflammatory bowel disease have demonstrated the safety, feasibility, and benefits of laparoscopic approach. ${ }^{[9]}$ Colon perforation is one of the complications of colonoscopy and treatment depends on the condition of the patient. Treatment can be either open or laparoscopic emergency surgery or conservative approach. The role of laparoscopy in patients with iatrogenic colonic perforation after the colonoscopy is important in terms of fewer complications, shorter hospital stay, and smaller incisions.

Our conversion rates in laparoscopic emergency colorectal surgery $(30 \%)$ were higher than in the literature. In the study performed by Masoomi et al., ${ }^{[10]}$ Laparoscopic surgery was found to be low in emergency patients, but conversion rate was $25 \%$. The wide variance of conversion rate is related to the patient selection, surgeon's experience, and procedure-related factors that affect the need for conversion in different studies. ${ }^{[11]}$ In our study, the reason for this increase was thought to be due to the flexibility of the patient selection criteria. Open surgery was required in 3 of 10 patients with mechanical bowel obstruction with air-fluid levels on plain abdominal Xray. Plain abdominal radiographs of those patients who were converted to open surgery (Fig. 1a, b) and completed laparoscopically (Fig. 2a, b) were shared with figures.

Our study was able to demonstrate that it could be safely performed in an emergency setting with acceptable perioperative results by experienced laparoscopists. Adequate lymph node collection, low mortality and morbidity, shorter hospital stay, and adequate surgical margins are possible when emergency laparoscopic colectomy is required for colon cancer.

\section{Conclusion}

Emergency laparoscopic colectomy in a well-selected patient group is feasible and safe when performed by experienced surgeons. The postoperative results are comparable with the open technique. Conversion rates are higher than elective surgery. Colonoscopy perforations and some colonic obstructions are suitable for the emergency laparoscopic approach.

\section{Disclosures}

Ethichs Committee Approval: The study was approved by the Local Ethics Committee.

Peer-review: Externally peer-reviewed.

Conflict of Interest: None declared.

\section{References}

1. Jacobs M, Verdeja JC, Goldstein HS. Minimally invasive colon resection (laparoscopic colectomy). Surg Laparosc Endosc 1991;1:144-50.

2. Agresta F, Campanile FC, Vettoretto N, Silecchia G, Bergamini C, Maida P, et al; Italian Surgical Societies Working Group. The Italian Surgical Societies Working Group Laparoscopic cholecystectomy: consensus conference-based guidelines. Langenbecks Arch Surg 2015;400:429-53. [CrossRef]

3. De Salvo GL, Gava C, Pucciarelli S, Lise M. Curative surgery for obstruction from primary left colorectal carcinoma: primary or staged resection? Cochrane Database Syst Rev 2004;2:CD002101. [CrossRef]

4. Frago R, Ramirez E, Millan M, Kreisler E, del Valle E, Biondo S. Current management of acute malignant large bowel obstruction: A systematic review. Am J Surg 2014;207:12738. [CrossRef]

5. Sauerland S, Agresta F, Bergamaschi R, Borzellino G, Budzynski A, Champault G, et al. Laparoscopy for abdom- 
inal emergencies: evidence-based guidelines of the European Association for Endoscopic Surgery. Surg Endosc 2006;20:14-29. [CrossRef]

6. Mauro MA, Koehler RE, Baron TH. Advances in gastrointestinal intervention: the treatment of gastroduodenal and colorectal obstructions with metallic stents. Radiology 2000;215:659-69. [CrossRef]

7. Huang X, Lv B, Zhang S, Meng L. Preoperative colonic stents versus emergency surgery for acute left-sided malignant colonic obstruction: a meta-analysis. J Gastrointest Surg 2014;18:584-91. [CrossRef]

8. De Ceglie A, Filiberti R, Baron TH, Ceppi M, Conio M. A meta-analysis of endoscopic stenting as a bridge to surgery versus emergency surgery for left-sided colorectal cancer obstruction. Crit Rev Oncol Hematol 2013;88:387-403. [CrossRef]

9. Aarons CB. Laparoscopic surgery for Crohn disease: a brief review of the literature. Clin Clin Colon Rectal Surg 2013;26:122-7. [CrossRef]

10. Masoomi H, Moghadamyeghaneh Z, Mills S, Carmichael JC, Pigazzi A, Stamos MJ. Risk factors for conversion of laparoscopic colorectal surgery to open surgery: does conversion worsen outcome? World J Surg 2015;39:1240-7. [CrossRef]

11. Moghadamyeghaneh $Z$, Masoomi H, Mills SD, Carmichael JC, Pigazzi A, Nguyen NT, et al. Outcomes of conversion of laparoscopic colorectal surgery to open surgery. JSLS 2014;18:e2014.00230. [CrossRef] 